PROCEEDINGS OF THE

AMERICAN MATHEMATICAL SOCIETY

Volume 131, Number 11, Pages 3345-3351

S 0002-9939(03)06920-X

Article electronically published on February 14, 2003

\title{
THE CLASS EQUATION AND COUNTING IN FACTORIZABLE MONOIDS
}

\author{
S. LIPSCOMB AND J. KONIECZNY
}

(Communicated by Stephen D. Smith)

\begin{abstract}
For orders and conjugacy in finite group theory, Lagrange's Theorem and the class equation have universal application. Here, the class equation (extended to monoids via standard group action by conjugation) is applied to factorizable submonoids of the symmetric inverse monoid. In particular, if $M$ is a monoid induced by a subgroup $G$ of the symmetric group $S_{n}$, then the center $Z_{G}(M)$ (all elements of $M$ that commute with every element of $G$ ) is $Z(G) \cup\{0\}$ if and only if $G$ is transitive. In the case where $G$ is both transitive and of order either $p$ or $p^{2}$ (for $p$ prime), formulas are provided for the order of $M$ as well as the number and sizes of its conjugacy classes.
\end{abstract}

\section{INTRODUCTION}

In this paper, we study monoids of restrictions of permutations under function composition. Indeed, for each subgroup $G$ of the symmetric group $S_{n}$ of all permutations of $N=\{1,2, \ldots, n\}$, we define the induced monoid

$$
M(G)=\{\alpha: \alpha \text { is a restriction of some } \sigma \in G\}
$$

whose multiplication is function composition. These monoids are inverse (for each $\alpha \in M(G)$, there is a unique $\beta \in M(G)$ satisfying $\alpha=\alpha \beta \alpha$ and $\beta=\beta \alpha \beta)$ and factorizable (each $\alpha \in M(G)$ factors $\alpha=\varepsilon \sigma$ where $\varepsilon$ is an idempotent and $\sigma \in G$ is a unit). In the special case $G=S_{n}$, the induced monoid $M\left(S_{n}\right)$ is denoted $C_{n}$, and it is widely known as the symmetric inverse monoid.

Abstract factorizable inverse monoids have been studied by Chen and Hsieh [1]. The definition of the property of being factorizable (given originally for inverse monoids) has been generalized to arbitrary semigroups: A semigroup $S$ is called factorizable if $S=G E$ for some subgroup $G$ of $S$ and some set $E$ of idempotents of $S$ [7]. For example, the semigroup $P T_{X}$ of all partial transformations on a set $X$ is factorizable if and only if $X$ is finite [7]. A similar result holds for the semigroup $T_{X}$ of all full transformations on $X[7]$ and the symmetric inverse semigroup $I_{X}$ of all partial one-to-one transformations on $X[1]$.

In the present paper, for various kinds of subgroups $G$ of $S_{n}$, we study the induced submonoids $M(G)$ of $C_{n}$. In Section 2 we provide definitions, in Section 3

Received by the editors April 12, 2002 and, in revised form, June 5, 2002.

2000 Mathematics Subject Classification. Primary 20M20, $20 \mathrm{M} 15$.

Key words and phrases. Factorizable monoids, symmetric inverse semigroups, class equation, conjugacy classes, permutation groups, transformation semigroups. 
we consider orders $|M(G)|$, and in Section 4, using the class equation for finite monoids, we investigate conjugacy classes.

\section{BASIC DEFINITIONS}

Let $M$ be a monoid with the identity 1 . Then $u \in M$ is a unit when $v \in M$ exists such that $u v=v u=1$. If such a $v$ exists, it is unique and denoted $u^{-1}$. The subset $G \subseteq M$ of all units is a subgroup of $M$, called the group of units of $M$. For example, $S_{n}$ is the group of units of $M\left(S_{n}\right)=C_{n}$. The monoid $C_{n}$ is of particular importance because every finite inverse monoid is isomorphic to a submonoid of $C_{n}$ for some $n$ [2, Theorem 5.1.7]. The elements of $C_{n}$ are partial one-to-one transformations on $N$, they are sometimes called charts [6], and the domain and range of the chart $\alpha$ are denoted, respectively, $\mathbf{d} \alpha$ and $\mathbf{r} \alpha$.

An element $b$ of a monoid $M$ is called an inverse of $a \in M$ if $a=a b a$ and $b=b a b$. When each $a \in M$ has exactly one inverse, then $M$ is an inverse monoid. For example, $C_{n}$ is an inverse monoid since the required unique inverse of $\alpha \in C_{n}$ is $\alpha^{-1}$.

Every inverse monoid $M$ has a natural partial order given by $a \leq b$ whenever an idempotent $e \in M$ exists such that $a=e b$ [2, p. 152]. An inverse monoid $M$ is factorizable when each $a \in M$ is "under" a unit $u \in M$, i.e., $a \leq u$ [4. In other words, an inverse monoid $M$ is factorizable if every element of $M$ can be factored as a product of an idempotent and a unit. For example, $C_{n}$ is factorizable: For each idempotent $\varepsilon \in C_{n}$ and each $x \in N$, either $x \varepsilon=x$ or $x \varepsilon$ is not defined, i.e., $\varepsilon$ is completely determined by its domain. So for $A \subseteq N, \varepsilon_{A}$ will denote the idempotent in $C_{n}$ with domain $A$. Next, for a unit $\sigma \in S_{n}$, the element $\varepsilon_{A} \sigma \in C_{n}$ is a restriction of $\sigma$ to $A$. Indeed, every $\alpha \in C_{n}$ is a restriction of some unit $\sigma \in S_{n}$ to $A=\mathbf{d} \alpha$. It follows that $C_{n}$ is a factorizable inverse monoid.

We shall use " $G \leq S_{n}$ " to indicate that $G$ is a subgroup of $S_{n}$. It is easy to see that the induced monoid $M(G)$ is an inverse submonoid of $C_{n}$. And since $M(G)$ consists of all elements of the form $\varepsilon \sigma$ where $\varepsilon \in C_{n}$ is idempotent and $\sigma \in G$, it is factorizable. In fact, $M(G)$ is the largest (with respect to inclusion) factorizable submonoid of $C_{n}$ that has $G$ as its group of units.

For $G \leq S_{n}$ and $A \subseteq N$, the stabilizer $G_{A}$ of $A$ consists of all $\sigma \in G$ such that $x \sigma=x$ for every $x \in A$. If $A=\{x\}$, we write $G_{A}=G_{x}$ and call $G_{x}$ the stabilizer of $x$. It is clear that $G_{A}$ is a subgroup of $G$. A subgroup $G \leq S_{n}$ is balanced if for all subsets $A, B \subseteq N$ of the same cardinality, we have $\left|G_{A}\right|=\left|G_{B}\right|$. For example, $S_{n}$ and its alternating subgroup $A_{n}$ are balanced, while the cyclic subgroup $G=\left\langle\left(\begin{array}{ll}1 & 2\end{array}\right)\right\rangle$ generated by the 2-cycle (12) in $S_{4}$ is not balanced. A group $G \leq S_{n}$ is transitive if for all $x, y \in N$, a $\sigma \in G$ exists such that $y=x \sigma$; semiregular if $G_{x}=\{1\}$ for each $x \in N$; and regular if it is both semiregular and transitive. Note that every semiregular group $G$ is balanced since $G_{A}=\{1\}$ for every non-empty $A \subseteq N$.

For abstract monoids $M$, we say $b \in M$ is conjugate to $a \in M$ if $b=u^{-1}$ au for some unit $u \in M$. The subset $[a]$ of all elements of $M$ conjugate to $a$ is the conjugacy class of $a$; and $\{[a]: a \in M\}$ is a partition of $M$.

For each set $A \subseteq N$, let $R_{A}^{M}=\left\{\varepsilon_{A} \sigma: \sigma \in G\right\}$ denote the set of all elements in $M=M(G)$ that have domain $A$. (The set $R_{A}^{M}$ is a Green's $\mathcal{R}$-class of $M$; see [2] p. 45] for the definition of Green's relations.) Note that if $A=\emptyset$, then $R_{A}^{M}=\{0\}$, where 0 is the empty (or zero) transformation on $N$. 


\section{ORDER OF $M(G)$}

The next lemma is crucial in proving the counting theorems of this section.

Lemma 3.1. Let $G \leq S_{n}$ and $M=M(G)$. For each $A \subseteq N$,

$$
\left|R_{A}^{M}\right|=\frac{|G|}{\left|G_{A}\right|} .
$$

Proof. Define $f: R_{A}^{M} \rightarrow G / G_{A}$ by $f\left(\varepsilon_{A} \sigma\right)=G_{A} \sigma$. Then $f$ is well-defined and one-to-one since for all $\sigma, \rho \in G$,

$$
\varepsilon_{A} \sigma=\varepsilon_{A} \rho \Leftrightarrow \varepsilon_{A}=\varepsilon_{A} \rho \sigma^{-1} \Leftrightarrow \rho \sigma^{-1} \in G_{A} \Leftrightarrow \rho \in G_{A} \sigma \Leftrightarrow G_{A} \sigma=G_{A} \rho .
$$

Since the function $f$ is clearly onto, it follows that $\left|R_{A}^{M}\right|$ is the number $|G| /\left|G_{A}\right|$ of right cosets of $G_{A}$ in $G$.

The next three theorems give, respectively, formulas for orders of monoids induced by balanced, semiregular, and transitive abelian groups. Let $k \in\{0,1, \ldots, n\}$. Then $C(n, k)$ denotes " $n$ choose $k$ " (the number of $k$-element subsets of $N$ ), and for a balanced group $G \leq S_{n}, m_{k}$ denotes $\left|G_{\{1, \ldots, k\}}\right|$. That is, $m_{k}$ is the size of the stabilizer $G_{A}$ for any $k$-element set $A \subseteq N$. Note that $G_{\emptyset}=G$ and so $m_{0}=|G|$.

Theorem 3.2. Let $G \leq S_{n}$ be balanced and $M=M(G)$. Then

$$
|M|=|G| \sum_{k=0}^{n} \frac{C(n, k)}{m_{k}} .
$$

Proof. When $A, B \subseteq N$ are distinct, $R_{A}^{M}$ and $R_{B}^{M}$ are disjoint (the domain of any $\varepsilon_{A} \sigma \in R_{A}^{M}$ is $\left.A\right)$. So for each $k \in\{0,1, \ldots, n\}$, there are $C(n, k)$ pairwise disjoint sets $R_{A}^{M}$ with $|A|=k$. But since $G$ is balanced, Lemma 3.1 shows that each set $R_{A}^{M}$ with $|A|=k$ has $|G| /\left|G_{A}\right|=|G| / m_{k}$ elements. Thus, $M$ has $C(n, k)\left(|G| / m_{k}\right)$ elements with domains of size $k$. The result follows.

To illustrate Theorem 3.2, first suppose $G=S_{n}$. Then $M=C_{n}$ and $m_{k}=$ $(n-k)$ ! for every $k$, showing that

$$
\left|C_{n}\right|=n ! \sum_{k=0}^{n} \frac{C(n, k)}{(n-k) !} .
$$

Second, suppose $G=A_{n}$. Then $M=A_{n}^{c}$, the alternating monoid. (The monoid $A_{n}^{c}$, which is a natural extension of the alternating group, was discovered by the first author [5].) For $G=A_{n}$, we have $m_{k}=1$ (if $k \geq n-1$ ) and $m_{k}=\frac{(n-k) !}{2}$ (if $k \leq n-2)$. Thus

$$
\left|A_{n}^{c}\right|=\frac{n !}{2} \sum_{k=0}^{n-2} \frac{C(n, k)}{\frac{(n-k) !}{2}}+\frac{n !}{2} C(n, n-1)+\frac{n !}{2} C(n, n)=n ! \sum_{k=0}^{n-2} \frac{C(n, k)}{(n-k) !}+\frac{(n+1) !}{2} .
$$

The next theorem is an immediate consequence of Theorem 3.2 because when $G$ is semiregular, it is balanced, $m_{0}=|G|$, and $m_{k}=1$ for each $k=1,2, \ldots, n$.

Theorem 3.3. Let $G \leq S_{n}$ be semiregular and $M=M(G)$. Then

$$
|M|=|G|\left(2^{n}-1\right)+1 .
$$


If $G \leq S_{n}$ is semiregular, then every non-zero $\alpha \in M(G)$ can be expressed uniquely as $\alpha=\varepsilon_{A} \sigma$ where $A=\mathbf{d} \alpha$ and $\sigma \in G$. Indeed, $\alpha=\varepsilon_{A} \sigma$ for some $\sigma \in G$ by the definition of $M(G)$. Suppose $\varepsilon_{A} \sigma=\varepsilon_{A} \delta$ where $\sigma, \delta \in G$. Then $\varepsilon_{A} \sigma \delta^{-1}=\varepsilon_{A}$ and so $\sigma \delta^{-1}$ fixes every $x \in A$. It follows that $\sigma \delta^{-1}=1\left(\right.$ since $A \neq \emptyset$ and $G_{x}=\{1\}$ for every $x \in N$ ), and so $\sigma=\delta$.

Note that the observation above provides another proof of Theorem 3.3: To construct a non-zero $\alpha=\varepsilon_{A} \sigma \in M(G)$, we have $2^{n}-1$ choices for $A$ and $|G|$ choices for $\sigma$.

For the next theorem, recall that the degree of $G \leq S_{n}$ is the number of points $x \in N$ such that $x \sigma \neq x$ for some $\sigma \in G$ (the number of points that are moved by $G)$. For example, the degree of $A_{n}$ is $n$ if $n>1$.

Theorem 3.4. Let $G \leq S_{n}$ be both transitive and abelian, and $M=M(G)$. Then

$$
|M|=|G|\left(2^{|G|}-1\right)+1
$$

Proof. Since $G$ is transitive and abelian, $G$ is regular [8. Proposition 4.4]. Thus, by Theorem 3.3, $|M|=|G|\left(2^{n}-1\right)+1$. Since $G$ is regular, its degree and order are equal [8. Proposition 4.2]. But the degree of $G$ is $n$ since a transitive group moves every point. Thus $n=|G|$ and so $|M|=|G|\left(2^{|G|}-1\right)+1$.

Corollary 3.5. Let $G \leq S_{n}$ be transitive, $M=M(G)$, and $p$ be a prime. Then:

(1) If $|G|=p$, then $|M|=p\left(2^{p}-1\right)+1$.

(2) If $|G|=p^{2}$, then $|M|=p^{2}\left(2^{p^{2}}-1\right)+1$.

Proof. The proof follows immediately from Theorem 3.4 and the fact that every group of order $p$ or $p^{2}$ is abelian.

\section{Conjugacy Classes}

Let $M$ be any monoid with $G$ its group of units. Then a singleton set $\{x\}$ is a conjugacy class if and only if $x$ commutes with every $u \in G$. It follows that the center

$$
Z_{G}(M)=\{x \in M: x u=u x \text { for every } u \in G\}
$$

of $M$ relative to $G$, which is a submonoid of $M$, counts the singleton conjugacy classes (just as the center $Z(G)$ of $G$ counts the singleton conjugacy classes in the group case). Moreover, $Z_{G}(M)$ is an inverse submonoid of $M$ when $M$ is an inverse monoid, and $Z_{G}(M)=Z(G)$ when $M=G$ is a group.

As in the group theory case, we use centralizers to count elements in nonsingleton conjugacy classes. For $x \in M$, we denote by $C_{G}(x)$ the centralizer of $x$ in $G$, which is defined as the set of all members of $G$ that commute with $x$. It is clear that the centralizer $C_{G}(x)$ is a subgroup of $G$.

For any finite group $G$, its class equation is

$$
|G|=|Z(G)|+\sum_{i}\left[G: C_{G}\left(a_{i}\right)\right]
$$

where one $a_{i} \in G$ is chosen from each conjugacy class in $G$ containing more than one element [3, $\left(42^{\prime}\right)$, p. 76$]$. The class equation for finite groups is a special case of the class equation for finite monoids. 
Theorem 4.1. Let $M$ be a finite monoid and let $G$ be its group of units. Then

$$
|M|=\left|Z_{G}(M)\right|+\sum_{i}\left[G: C_{G}\left(x_{i}\right)\right]
$$

where one $x_{i} \in M$ is chosen from each conjugacy class in $M$ containing more than one element.

Proof. By the definition of $Z_{G}(M),\left|Z_{G}(M)\right|$ is the number of singleton conjugacy classes. Let $x \in M$ be chosen from a conjugacy class $[x]$ containing more than one element, and let $H=C_{G}(x)$. It suffices to show that the number $|[x]|$ of conjugates of $x$ is equal to $[G: H]$. To this end, define $f:[x] \rightarrow G / H$ by $f\left(u^{-1} x u\right)=H u$. The function $f$ is well-defined and one-to-one since

$$
u^{-1} x u=v^{-1} x v \Leftrightarrow x u v^{-1}=u v^{-1} x \Leftrightarrow u v^{-1} \in C_{G}(x)=H \Leftrightarrow H u=H v .
$$

Since $f$ is clearly onto, $|[x]|=[G: H]$.

Note that the class equation (1) is a special case of a more general formula enumerating the elements of a finite set $S$ on which an action of a finite group $G$ is defined [3, (40), p. 76]. In particular, the action underlying Theorem 4.1 is that of $G$ acting on $M$ by conjugation.

Corollary 4.2. Let $M$ be a finite monoid and let $G$ be its group of units. Then the order of each conjugacy class of $M$ divides the order of $G$.

Proof. Let $x \in M$. By the proof of Theorem 4.1, $|[x]|$ is equal to $\left[G: C_{G}(x)\right]$, which divides $|G|$.

In passing, we observe that for any $u \in G$, we have $x \in G$ if and only if $u^{-1} x u \in$ $G$. It follows that each conjugacy class of $M$ is either a subset of $G$ or of $M-G$. In other words, the set of conjugacy classes of a finite monoid refines the doubleton partition $\{G, M-G\}$ of $M$.

We shall now investigate the center $Z_{G}(M)$ of a submonoid $M$ of $C_{n}$ induced by its group of units $G$. The next theorem shows that only special elements of a monoid $M$ induced by $G \leq S_{n}$ can be members of $Z_{G}(M)$. A subset $A \subseteq N$ is called invariant under $G$ if $A \sigma \subseteq A$ for every $\sigma \in G$.

Theorem 4.3. Let $G \leq S_{n}$ and $M=M(G)$. Let $\alpha \in Z_{G}(M)$ with $A=\mathbf{d} \alpha$. Then $A$ is invariant under $G$ and $\alpha$ is a permutation on $A$.

Proof. Since $A=\mathbf{d} \alpha$, we have $\alpha=\varepsilon_{A} \sigma$ for some $\sigma \in G$. Let $\rho$ be any element of $G$. Since $\alpha \in Z_{G}(M)$, we have $\varepsilon_{A} \sigma \rho=\rho \varepsilon_{A} \sigma$. Let $x \in A$. Then $x\left(\varepsilon_{A} \sigma \rho\right)$ is defined, and so $x\left(\rho \varepsilon_{A} \sigma\right)$ is also defined. But the latter can happen only if $x \rho \in A$. Thus $A \rho \subseteq A$ and so $A$ is invariant under $G$. In particular, $A \sigma \subseteq A$ and so $\alpha=\varepsilon_{A} \sigma$ is a permutation on $A$.

For any $G \leq S_{n}$ and $M=M(G)$, we have $Z(G) \cup\{0\} \subseteq Z_{G}(M)$ where 0 is the zero transformation on $N$. The next theorem characterizes those groups $G$ for which $Z_{G}(M)=Z(G) \cup\{0\}$, that is, $Z_{G}(M)$ is as small as possible.

Theorem 4.4. Let $G \leq S_{n}$ and $M=M(G)$. Then $Z_{G}(M)=Z(G) \cup\{0\}$ if and only if $G$ is transitive. 
Proof. Suppose $G$ is transitive and $\alpha=\varepsilon_{A} \sigma \in Z_{G}(M)-(Z(G) \cup\{0\})$. Then $A$ is a non-empty proper subset of $N$. Choose $x \in A$ and $y \in N-A$. Since $G$ is transitive, there is $\rho \in G$ such that $x \rho=y$. Then $\alpha \rho=\rho \alpha$ implies that $x\left(\varepsilon_{A} \sigma \rho\right)=x\left(\rho \varepsilon_{A} \sigma\right)=y\left(\varepsilon_{A} \sigma\right)$. But this is a contradiction since $x\left(\varepsilon_{A} \sigma \rho\right)$ is defined and $y\left(\varepsilon_{A} \sigma\right)$ is undefined.

Conversely, suppose $G$ is not transitive. Then for a fixed $x \in N$, let $A \subseteq N$ be the $G$-orbit of $x$, that is, $A=\{x \sigma: \sigma \in G\}$. Since $G$ is not transitive, $A$ is a proper subset of $N$, showing that the idempotent $\varepsilon_{A} \notin G \cup\{0\}$. In addition, since $A \sigma=A$ for each $\sigma \in G$, we have $\varepsilon_{A} \sigma=\sigma \varepsilon_{A}$ for each $\sigma \in G$. Thus $\varepsilon_{A} \in Z_{G}(M)$ and so $Z_{G}(M) \neq Z(G) \cup\{0\}$.

For applications of the results above, we provide two theorems.

Theorem 4.5. Let $G \leq S_{n}$ be transitive of order $p$, where $p$ is a prime, and let $M=M(G)$. Then $M$ has $p+1$ singleton conjugacy classes and $2^{p}-2$ non-singleton classes, each of size $p$.

Proof. Since $G$ is transitive and abelian, Theorem 4.4 yields $Z_{G}(M)=Z(G) \cup\{0\}=$ $G \cup\{0\}$. Thus $M$ has $|G|+1=p+1$ singleton conjugacy classes. For a nonsingleton class $[\alpha]$, Corollary 4.2 shows that $|[\alpha]|$ divides $|G|=p$. Thus, every such class has size $p$. It follows that the number $m$ of non-singleton classes satisfies $p+1+m p=|M|$. But this equation coupled with $|M|=\left(2^{p}-1\right) p+1$ (Corollary 3.5) shows that $m=2^{p}-2$.

Theorem 4.6. Let $G \leq S_{n}$ be transitive of order $p^{2}$, where $p$ is a prime, and let $M=M(G)$. Then $M$ has $p^{2}+1$ singleton conjugacy classes and $2^{p^{2}}+(q-1) 2^{p}-2 q$ non-singleton classes where $q=p$ when $G$ is cyclic and $q=p^{2}$ otherwise. Moreover, each non-singleton class is either of size $p$ or size $p^{2}$ :

(1) If $G$ cyclic, $M$ has $p\left(2^{p}-2\right)$ classes of size $p$ and $2^{p^{2}}-2^{p}$ of size $p^{2}$.

(2) Otherwise, $M$ has $p(p+1)\left(2^{p}-2\right)$ classes of size $p$ and $2^{p^{2}}-(p+1) 2^{p}+2 p$ of size $p^{2}$.

Proof. Since $G$ is transitive, $Z_{G}(M)=Z(G) \cup\{0\}$ (Theorem 4.4), and since $G$ is abelian, $Z(G)=G$. Thus $M$ has $|G|+1=p^{2}+1$ singleton conjugacy classes. For a non-singleton class $[\alpha]$, Corollary 4.2 shows that $|[\alpha]|$ divides $|G|=p^{2}$. Thus, every such class is either of order $p$ or $p^{2}$. In addition, if $|[\alpha]|=p$, then $\left[G: C_{G}(\alpha)\right]=p$ (proof of Theorem 4.1), and so $\left|C_{G}(\alpha)\right|=p$.

Suppose $G=\langle\tau\rangle$ is cyclic. First, we count $\alpha \in M$ such that $\left|C_{G}(\alpha)\right|=p$, that is, $C_{G}(\alpha)=\left\langle\tau^{p}\right\rangle$, the unique subgroup of $G$ of order $p$. Thus it suffices to count those $\alpha \in M-Z_{G}(M)$ that commute with $\tau^{p}$. Note that $\tau^{p}$ is a product $\tau^{p}=\rho_{1} \rho_{2} \ldots \rho_{p}$ of $p$ disjoint cycles, each of length $p$. By the observation after Theorem 3.3, $\alpha$ can be expressed uniquely as $\alpha=\varepsilon_{A} \sigma$, where $A \subseteq N$ and $\sigma=\tau^{k} \in G$ for some $k=1,2, \ldots, p^{2}$. We have that $\alpha$ commutes with $\tau^{p}$, that is, $\varepsilon_{A} \tau^{k} \tau^{p}=\tau^{p} \varepsilon_{A} \tau^{k}$. Multiplying both sides of the last equality by $\tau^{-k}$, we obtain $\varepsilon_{A} \tau^{p}=\tau^{p} \varepsilon_{A}$. It follows that $\alpha=\varepsilon_{A} \sigma$ and $\tau^{p}$ commute precisely when $A$ is the set of all points in $N$ moved by some subset of cycles in the cycle decomposition of $\tau^{p}$.

Now, the number of elements of $M-Z_{G}(M)$ of the form $\varepsilon_{A} \sigma$, where $A$ is the set of all points in $N$ moved by some subset of cycles in the cycle decomposition of $\tau^{p}$, and $\sigma \in G$, is $\left(2^{p}-2\right) p^{2}$ (since we have $2^{p}-2$ choices for $A$ and $p^{2}$ choices for $\sigma$ ). Hence the number of conjugacy classes in $M$ with $p$ members is $\frac{\left(2^{p}-2\right) p^{2}}{p}=p\left(2^{p}-2\right)$. 
Turning to the number of conjugacy classes in $M$ with $p^{2}$ members, we let $m$ denote the number of classes of size $p^{2}$. Then, recalling the formula for the size of $M$ (Corollary 3.5), we have

$$
\left[p^{2}+1\right] \cdot 1+\left[p\left(2^{p}-2\right)\right] \cdot p+m \cdot p^{2}=p^{2}\left(2^{p^{2}}-1\right)+1
$$

which implies $m=2^{p^{2}}-2^{p}$.

Next, we suppose that $G$ is not cyclic. Again, we count $\alpha \in M$ such that $\left|C_{G}(\alpha)\right|=p$. Since $G$ is not cyclic, every non-identity element of $G$ has order $p$. It follows that $G$ has exactly $p+1$ subgroups of order $p$, say $H_{1}, \ldots, H_{p+1}$, with $H_{i} \cap H_{j}=\{1\}$ for $i \neq j$. For $i \in\{1, \ldots, p+1\}$, let $\tau_{i}$ be a generator of $H_{i}$. For every $\alpha \in M,\left|C_{G}(\alpha)\right|=p$ if and only if $C_{G}(\alpha)=H_{i}$ for some $i=1, \ldots, p+1$. Thus it suffices to count those $\alpha \in M-Z_{G}(M)$ that commute with $\tau_{i}$ for exactly one $i \in\{1, \ldots, p+1\}$.

Fix $\tau_{i}$ and note that, since $G$ is regular and $n=p^{2}, \tau_{i}$ is a product $\tau_{i}=\rho_{1} \rho_{2} \cdots \rho_{p}$ of $p$ disjoint cycles, each of length $p$. By the argument in the cyclic case above, the number of $\alpha \in M-Z_{G}(M)$ that commute with the fixed $\tau_{i}$ is $\left(2^{p}-2\right) p^{2}$. Thus the number of $\alpha \in M-Z_{G}(M)$ that commute with exactly one $\tau_{i}$ is $(p+1)\left(2^{p}-1\right) p^{2}$ (since we have $p+1$ choices for $\tau_{i}$ ). Hence the number of conjugacy classes in $M$ with $p$ members is $\frac{\left(2^{p}-2\right)(p+1) p^{2}}{p}=p(p+1)\left(2^{p}-2\right)$.

Turning to the number of conjugacy classes in $M$ with $p^{2}$ members, we let $m$ denote the number of classes of size $p^{2}$. Then, recalling the formula for the size of $M$ (Corollary 3.5), we have

$$
\left[p^{2}+1\right] \cdot 1+\left[p(p+1)\left(2^{p}-2\right)\right] \cdot p+m \cdot p^{2}=p^{2}\left(2^{p^{2}}-1\right)+1
$$

which implies $m=2^{p^{2}}-(p+1) 2^{p}+2 p$.

\section{REFERENCES}

[1] S.Y. Chen and S.C. Hsieh, Factorizable inverse semigroups, Semigroup Forum 8 (1974), 283-297. MR 51:13089

[2] J.M. Howie, Fundamentals of Semigroup Theory, Oxford University Press, New York, 1995. MR 98e:20059

[3] N. Jacobson, Basic Algebra I, W.H. Freeman Company, New York, 1985. MR 86d:00001

[4] M.V. Lawson, Inverse Semigroups, World Scientific, Singapore, 1998. MR 2000g:20123

[5] S.L. Lipscomb, Problems and applications of finite inverse semigroups, Words, languages and combinatorics (Kyoto, 1990), 337-352, World Sci. Publishing, River Edge, NJ, 1992. MR 93a:20104

[6] S.L. Lipscomb, Symmetric Inverse Semigroups, Mathematical Surveys and Monographs, vol. 46, American Mathematical Society, Providence, RI, 1996. MR 97j:20065

[7] Y. Tirasupa, Factorizable transformation semigroups, Semigroup Forum 18 (1979), 15-19. MR 80j:20072

[8] H. Wielandt, Finite Permutation Groups, Academic Press, New York, 1964. MR 32:1252

Department of Mathematics, Mary Washington College, Fredericksburg, Virginia 22401

E-mail address: slipscom@mwc.edu

Department of Mathematics, Mary Washington College, Fredericksburg, Virginia 22401

E-mail address: jkoniecz@mwc.edu 